\title{
Risk Factors for Peyronie's Disease in Patients Submitted to the Nesbit Procedure
}

\author{
Thiago da Silveira Antoniassi ${ }^{1}$, Luis César Fava Spessoto ${ }^{1}$, José \\ Roberto Gonçalves Filho ${ }^{1}$, Vinícius Matias Monteiro Cavalcante ${ }^{1}$, \\ Etore Andrade da Silva1, Maria Fernanda W. Facio', Laisa Ferraz \\ de Arruda1', Jerônimo Ferraz de Arruda Neto ${ }^{1}$, José Germano F. \\ Arruda1 ${ }^{1}$, Germano José F. Arruda ${ }^{1}$, Marcio Gatti ${ }^{1}$, Pedro Francisco \\ F. Arruda1, Fernando Nestor Facio Junior ${ }^{1}$
}

1Discipline of Urology, Department of Surgical specialties, Medical School of Sao Jose do Rio Preto (FAMERP) School of Medicine (FACERES)

School of Medicine (UNILAGO)

\begin{abstract}
Peyronie's disease is a benign disease of unknown etiology. The objective of this study was to evaluate factors associated to Peyronie's disease in patients submitted to the Nesbit surgical procedure. Patients and We conducted a retrospective study in 67 patients through a descriptive analysis of patients submitted to the Nesbit surgical procedure in Hospital de Base in Sao Jose do Rio Preto. Results: The main factors associated with Peyronie's disease found in this study were smoking (46.2\%), hypertension (41.7\%) and excessive alcohol consumption (25.3\%). Most patients took some type of medication daily, in particular antihypertensive drugs. Significant associations were found with smoking, hypertension and alcohol abuse.
\end{abstract}

Keywords: antihypertensive drugs, Peyronie's disease, risk factors

\begin{abstract}
Introduction
Peyronie's disease is a benign urological disease of unknown etiology. It is a healing disorder after repetitive injuries (microtraumas) with the formation of inelastic areas of tunica albuginea potentially leading to the formation of typical symptoms of penile plaque: penile pain, curvature and erectile dysfunction (Hauptmann et al., 2011).
\end{abstract}

In most cases, the onset is associated with an acute phase, consisting of painful erections, palpable plaque and curvature of the penis. Pain can be controlled with analgesics and the tortuosity process stabilizes after between 12 and 18 months. A secondary quiescent phase follows characterized by stable deformity and lack of pain with healing (Graziottin et al., 2001).

The plaque is usually located on the dorsal surface of the penis with a corresponding dorsal penile deformity. Lateral and ventral plaques are not as common, but result in greater difficulty for intercourse. It has been reported that this occurs in association with Dupuytren's contractures, plantar fascia contractures, tympanosclerosis, trauma, urethral instrumentation, diabetes mellitus, uric gout, Paget's disease and the use of beta-blockers (Carrieri et al., 1998).

\begin{abstract}
Rhoden et al. (2001) showed a prevalence in over 50year-old men of around 14\%, with most patients being Caucasian $(88 \%)$. This study also found a rate of erectile dysfunction in patients with Peyronie's disease of approximately $68 \%$. According to the literature, the main risk factor related to the onset of this disease is trauma (Christopher et al., 2005). Moreover, the few studies that demonstrate the direction of penile deformity report that the most common curvature is dorsal (Kadioglu et al., 2011). Recent epidemiological studies have reported a prevalence of Peyronie's disease in between 3-5\% of the general male population with two-thirds of the
\end{abstract}

This article is published under the terms of the Creative Commons Attribution License 4.0

Author(s) retain the copyright of this article. Publication rights with Alkhaer Publications.

Published at: http://www.ijsciences.com/pub/issue/2016-06/

DOI: 10.18483/ijSci.1073; Online ISSN: 2305-3925; Print ISSN: 2410-4477 
patients aged between 40 and 60 years (Sasso et al., 2007).

Among the differential diagnoses are congenital curvature of the penis, chordee with or without hypospadias, dorsal venous thrombosis of the penis, cavernous fibrosis secondary to local trauma, leukemic infiltration of the corpora cavernosa, ventral curvature secondary to urethral instrumentation, primary or secondary benign or malignant tumors, late syphilitic lesions and infiltration of lymphogranuloma venereum (Graziottin et al., 2001).

The initial approach to treatment should be conservative as the lesion may progress, stabilize or even regress. Oral agents commonly used empirically are vitamin E, potassium aminobenzoate (Potaba), tamoxifen and colchicine. Intralesional injectable drugs such as interferon, verapamil and more recently Clostridium histolyticum collagenase, the first treatment approved by the US Food and Drug Administration (FDA), can be used (Hellstrom et al., 2009). Indications for surgical treatment include severe curvature, notching deformity or thinning of the penile over more than one year, difficulty in sexual intercourse, the partner's discomfort due to the deformity, or severe penile shrinkage (Hauptmann et al., 2011).

Recent studies have tried to establish risk factors for Peyronie's disease as this could help therapeutic management. The knowledge of the pathogenesis and natural history of the disease would allow the patient and his partner to adopt a conscious decision about treatment options and expected results.
The objective of this study was to evaluate factors associated with Peyronie's disease in patients submitted to the Nesbit procedure in a general hospital.

\section{Patients and Methods}

This study was a retrospective analysis of medical records of patients submitted to penile plication surgery using the Nesbit procedure between 1 January 2005 and 31 December 2015. All surgeries were performed in Hospital de Base of the Medical School in São José do Rio Preto (FAMERP), SP, Brazil. This study was approved by the Research Ethics Committee of FAMERP.

Sixty-seven patients were submitted to this procedure during the 11 years of this study. Data were collected and tabulated from the patients' records in respect to age, race, trauma, medications taken and type of tortuosity. Statistical analysis was performed to investigate possible risk factors for Peyronie's disease and characteristics of this patient population.

\section{Results}

The ages of the patients in this study ranged from 20 to 86 years with a mean age of 58 years; most patients were aged between 52 and 68 years.

Among the main factors associated with Peyronie's disease, the most common were smoking (46.2\%), hypertension $(41.7 \%)$ and excessive alcohol consumption $(25.3 \%)$ (Table 1$)$.

Although trauma is identified as the main initial factor of Peyronie's disease, we found a low correlation $(2.9 \%)$ in this study.

Table 1 - Risk factors related to Peyronie's disease

\begin{tabular}{lll}
\hline Risk factor & $\mathrm{n}$ & $\%$ \\
\hline smoking & 31 & 46.2 \\
hypertension & 28 & 41.7 \\
high alcohol ingestion & 17 & 25.3 \\
dyslipidemia & 10 & 14.9 \\
diabetes & 6 & 8.9 \\
trauma & 2 & 2.9 \\
\hline
\end{tabular}

Peyronie's disease is more prevalent among Caucasians (86.5\%) than among Blacks (13.4\%). This study found a dorsal predominance for the direction of the deformity $(38.8 \%)$ as reported in other studies, followed by a left curvature $(23.9 \%)$, and an association of these two malformations (16.4\%) (Table 2). 
Table 2 - Direction of the tortuosity

\begin{tabular}{lll}
\hline Direction & $\mathrm{N}$ & $\%$ \\
\hline dorsal & 26 & 38.8 \\
to left & 16 & 23.9 \\
ventral & 8 & 11.9 \\
to right & 1 & 1.5 \\
dorsal + left & 11 & 16.4 \\
dorsal + right & 2 & 3 \\
ventral + left & 3 & 4.5 \\
ventral + right & 0 & 0 \\
\hline Total & 67 & 100 \\
\hline
\end{tabular}

Most patients (50.7\%) were taking medications for chronic conditions, in general more than two types of drugs on a daily basis. Of the 34 patients who were taking medications, 28 (82.3\%) were taking antihypertensive drugs

\section{Discussion}

There are few published studies about risk factors for Peyronie's disease. This study describes the prevalence of various factors that may be involved in the pathology of this disease in patients seeking treatment using penile plication surgery in a university hospital. More than two centuries after its first description, the etiology of Peyronie's disease remains unclear but the most accepted hypothesis for its onset is penile trauma (Sommer et al., 2002). The current study shows that trauma was responsible in few patients $(2.9 \%)$. This may be explained by the fact that patients often do not report microtraumas that they may have suffered.

The main factors found associated with Peyronie's disease in this study were smoking, high blood pressure and excessive alcohol consumption. These factors may be responsible for causing vascular fragility resulting in bruising and consequently fibrotic plaque corroborating the theory of the probable genesis of Peyronie's disease (Muralidhar et al., 1997). The age distribution is consistent with other series of patients reported in the literature, which state that most patients are between 45 and 86 years with an average age of approximately 55 years (Levine et al., 2003). This study showed that the majority of patients were in this age group, but few were older than 76 years old.

Of the patients taking some type of medication, the use of antihypertensive drugs (82.3\%) stood out; the most common of these drugs was angiotensinconverting enzyme inhibitors $(50 \%)$. There are plenty of studies linking the use of beta-blockers with Peyronie's disease, but there are no reports related to other antihypertensive agents (Muralidhar et al., 1997; Tunuguntla et al., 2001; Paladini et al., 1981).

The most prevalent direction of penile curvature was in line with published studies (Kadioglu et al., 2011). As the current study was retrospective, it was impossible to evaluate the degree of curvature only the direction.

\section{Conclusions}

This study sought to identify possible risk factors associated with Peyronie's disease. It found significant associations with smoking, hypertension and alcohol abuse with the use of drugs, especially antihypertensive drugs, being present in many patients with the disease thereby suggesting a close correlation. Prospective, randomized, controlled studies should be conducted to confirm these findings.

\section{References}

1) Hauptmann A, Diemer T, Weidner W. Peyronie's disease: diagnostic and therapy Urologe A 2011;50(5):609-20.

2) Graziottin TM, Resplande J, Gholami SS, Lue TF. Peyronie's disease Braz J Urol 2001;27:326-340.

3) Carrieri MP, Serraino D, Palmiotto F, Nucci G, Sasso F. A case-control study on risk factors for Peyronie's disease. J Clin Epidemiol. 1998;51: 511-515.

4) Rhoden E L, Teloken C, Ting H Y, Lucas M L, Ros C T, Souto VCA. Prevelence of Peyronie's Disease in men over 50-y-old from southern Brazil. Int J Impot Res 2001; 13(5): 291-3.

5) Christopher JS, Chelsea M, Ridwan S. Peyronie's disease: the epidemiology, aetiology and clinical evaluation of deformity. BJU Int 2005; 95:729-32.

6) Kadioglu A, Sanli O, Akman T, Canguven O, Aydin M, Akbulut F, Kucukdurmaz F. Factors affecting the degree of penile deformity in Peyronie's disease: an analysis of 1001 patients. J Androl 2011;32(5):502-8.

7) Sasso F, Gulino G, Falabella R, D’Adessi A, Sacco E, D'Onofrio A, Bassi PF. Peyronie's disease: lights and shadows. Urol Int 2007;78 1-9.

8) Hellstrom WJ. Medical management of Peyronie's disease. J Androl 2009;30(4):397-405.

9) Sommer F, Schwarzer U, Wassmer G, Bloch W, Braun M, Klotz T, Engelmann U. Epidemiology of Peyronie's disease Int J Impot Res 2002;14(5):379-83.

10) Muralidhar S, Kumar B, Sharma SK, Sharma M, Mandal AK. Etiologic factors in Peyronie's disease Int $\mathrm{J}$ Dermatol 1997;36(8):579-81.

11) Levine LA, Estrada CR, Storm DW, Matkov TG. Peyronie's disease in younger men: characteristics and treatment results. J Androl 2003;24(1):27-32.

12) Tunuguntla HS. Management of Peyronie's disease: a review. World J Urol 2001;19(4):244-50.

13) Paladini G. Peyronie's disease and systemic lupus erythematous syndrome associated with metoprolol administration: a case report. Int $\mathrm{J}$ Tissue React $1981 ; 3(2): 95-8$ 